Research Article

\title{
Statistical Calculation Method and Analysis of Athletes' Biorhythm State and Sports Injury
}

\author{
Shaohui Su iD \\ Physical Education Department, Bengbu University, Bengbu 233030, Anhui, China \\ Correspondence should be addressed to Shaohui Su; ssh@bbc.edu.cn
}

Received 30 April 2021; Revised 21 May 2021; Accepted 2 June 2021; Published 16 June 2021

Academic Editor: Sang-Bing Tsai

Copyright (C) 2021 Shaohui Su. This is an open access article distributed under the Creative Commons Attribution License, which permits unrestricted use, distribution, and reproduction in any medium, provided the original work is properly cited.

\begin{abstract}
In recent years, attention has been focused on the prevention and treatment of sports injuries. However, no athlete injury prevention system has been established. Athletes' injury prevention has become an important research field, but it is still used in current sports injury statistics. Traditional calculation methods are difficult to meet the requirements of modern halo. In order to understand the relationship between the athlete's biorhythm state and the statistical calculation method of sports injury and to reduce the damage caused by sports injury to athletes, we have carried out statistics on various physical parameters of athletes in a certain university in this city through example analysis and creatively introduced the statistical calculation method of the fault tree, and the corresponding results provide a certain research foundation for the subsequent research. The research results in this article prove that formulating a suitable sport mode for athletes can improve their athletic ability by more than $10 \%$, reduce damage to athletes, and extend their professional life. Generally speaking, athletes' injuries can be reduced by more than $15 \%$. This shows that appropriate injury statistics calculation methods and research on sports injury prevention in advance are extremely important for athletes.
\end{abstract}

\section{Introduction}

As the main body of competitive sports, the country has invested a lot of mental, human, and financial resources to train an outstanding athlete. Athletes have put their precious youth and effort into good results and have reflected their social value through their competitiveness. [1]. The development of competitive sports athletes and the improvement of competitive sports levels may be restricted due to sports injuries, hindering the career development of athletes. Therefore, having a healthy physical condition is one of the important reasons for athletes to extend their sports career life. The prevention and rehabilitation of sports injuries is very important [2].

Biological rhythm is one of the basic characteristics endowed to life by natural evolution. Humans and all living things must be controlled and influenced by biological rhythms. Human biological rhythms refer to physical rhythms, emotional rhythms and intellectual rhythms [3]. Once an athlete suffers a sports injury, it will affect the improvement of training and competition performance and may lead to a decline in the overall competitive level of the team. Therefore, having the right awareness and concept of sports injury prevention can reduce the incidence of sports injuries and extend the life of athletes. Through the study of biological rhythms, we can effectively know the changes of various functions of the athletes during exercise, effectively prevent various sports injuries, and protect the athletes [4].

For the statistical calculation methods and analysis of sports injuries, experts at home and abroad have always done many research studies. In foreign countries, sports have emerged earlier, and the research on sports injuries has been more complete. Forsdyke et al. fixed the sensor device on the back of the basketball player's hand and collected the acceleration and angular velocity data of the player's hand during the jump shot. The jump shot process is divided into four stages, the shooting posture of each stage is analyzed, and voice feedback is used to remind the athletes to correct the shooting posture. However, the athlete's jump shot posture is reflected by the motion posture of his arms and 
legs. This method only analyzes the posture of the hands during the shooting process and lacks consideration of the posture of other parts of the body [5]. Paul and others collected the athletes' heart rate, oxygen consumption, and acceleration data, analyzed the physiological characteristics of basketball players during exercise, compared the physiological performance of athletes in different forms of competition and training, and identified the parts that may cause sports injuries [6]. Domestic sports injury research started late. Forsdyke et al. pointed out that, with the improvement of the country's overall strength, quality education has become the basis for talent training. The comprehensive development of students' physical and mental quality is becoming more and more important to the development of a country. The cognitive level of injury is not enough, and the statistical method for sports injury is also difficult to meet, so a new statistical calculation method is required [5]. Everhart et al. pointed out that badminton is an antagonistic sport across the net, especially doubles, which requires the tacit cooperation of the two. Therefore, in college badminton teaching sports injuries, new teaching methods should be tried to avoid students' sports injuries [7]. These studies have provided some references for the research of this article, but due to the lack of samples in previous experiments, the experimental results are difficult to reproduce and are not operable.

The innovation in this article is to address issues such as athlete modeling and sports strategy choices; regarding the location and type of injury in various sports, the design is based on biological rhythm state theory, theory of multiple intelligence, project reflex theory, and fault tree technology; to describe the theoretical model structure of the state parameters of different athletes; to get a new method for comprehensive evaluation of athletes' sports injuries; to use the constructed structural equation for comprehensive evaluation to analyze the relative importance of various indicators of athletes; and to conclude with the determination of sports injury factor.

\section{Statistical Calculation Method of Athletes' Biorhythm Status and Sports Injury}

2.1. Sports. Sports itself has its own unique characteristics: physical activity is used as a means to exercise the body, enhance physical fitness, and promote the overall development of the human body [7]. In the process of social development, sports are restricted to a certain extent by political and economic factors and in turn provide services for politics and economy. The true meaning of sport took shape very early on. In other words, it is a primitive form of competition for the purpose of winning [8]. Since then, the content of this form of sports activities has become more colorful, and many competitive sports have initially taken shape, laying the foundation for the development of modern sports. In the field of modern sports development, competition activities have gained greater and greater independence and have been named "competitive sports." It is necessary to strengthen the basic technical training of athletes. There are many basic skills of athletes, and the training of basic dribbling is particularly important. Only by mastering accurate basketball dribbling can the techniques and tactics be fully utilized $[6,9]$.

It is very important to ensure the standardization and accuracy of basic movements. In order to ensure the standardization of basic movements, a coach must be supervised and guided to provide real-time guidance and advice to athletes [10]. This kind of manual guidance takes a lot of time and complexity for the coaches. Too high intensity is easy to cause damage to athletes, and it is difficult to achieve accurate estimation of exercise intensity at present.

2.2. Athlete Injury and Its Detection. There are many reasons for sports injuries, such as poor self-protection awareness, insufficient preparation activities, incorrect technical movements, and poor physical condition; external factors include venue facilities, intentional or unintentional fouls by opponents, etc. [11]. Among them, own factors account for a larger proportion. Whether in training or competition, sports injuries happen by chance, and most sports injuries are unpredictable, so how to reduce sports injuries becomes the key. The action mode is the process of executing the corresponding action program stored in the brain by muscles, fascia, and joints under the control of the central nervous system. This execution process is carried out in a certain time and space sequence [12].

Sports injuries are generally divided into acute and chronic injuries and are discussed. Acute injury refers to immediate damage to body tissue or damage that causes symptoms caused by sudden external forces. The common types of acute injury are divided into the following categories. (1) Sprain: the ligament is torn or damaged due to twisting or overextension [13]. In severe ligament sprains, severe hematomas begin to occur due to the rupture of capillaries, and the injured area will be painful and swollen, causing inability to move. (2) Strain: abnormal muscle activity or sudden huge contraction can cause muscle fiber breakage; slight strain may be caused by the breakage of only a small part of the muscle fibers, and there is no obvious change in appearance. Only when the injured part is pressed hard or pressed, will the vine pain be felt; and severe muscle strain may be caused by the complete breakage of the muscle fibers. The junction between muscles and infrastructure is separated. (3) Bruises: it often occurs in sports that require close contact [14]. Bone or muscle is impacted by external force, causing microvascular rupture and bleeding and muscle fiber rupture, and internal bleeding in the injured area. If not properly treated, the injured area may develop tissue fibrous calcification accompanied by chronic painful conditions. (4) Fractures: bone fatigue caused by overtraining or broken bones caused by external force impact can be divided into open fractures and closed fractures. The injured part may be swollen due to internal bleeding and may be deformed and distorted in appearance. A serious condition may lead to shock. (5) Joint dislocation [11]: the joint capsule ruptured and the joint bones shifted due to the impact of external force. The appearance is clearly distorted, and rupture or excessive extension of the ligaments can 
cause bleeding or swelling where the joint has fallen off. If you have experience of joint dislocation, frequent injuries to the same part are likely to cause ligament laxity and joint capsule expansion and evolve into habitual dislocation [15]. Chronic injury refers to the long-term accumulation of multiple small injuries, which may be insensitive or slightly sensible, and it is easy to be negligent. The injured may not be discovered until it affects sports performance or has physical discomfort. In addition, the injured part may be transformed into chronic sports injury after acute injury.

There are five common chronic sports injuries [16]. (1) Arthritis: due to the excessive load of the joints, the joints are damaged and degenerative diseases occur. There are often noises when the joints in the injured area move, there will be pain and tenderness, or there will be the phenomenon of inability to exert force. (2) Tendinitis: most of it occurs near the joints. Due to long-term overuse of muscles, the tendons become chronically inflamed, causing swelling, soreness, and weakness. (3) Fatigue fractures: due to overuse, the bone cannot withstand the large pressures after long periods of repeated operation and pressure fractures form at the pressure points. There is persistent and acute pain in the injured area, mainly the tibia. (4) Secondary injuries: the second-degree injury may be caused by the injury site not receiving proper rehabilitation and failing to obtain a good recovery. (5) Ligament injury: because of the accumulation of minor injuries, the function of ligaments decreases [17].

When the body is injured, the reaction of local tissues may cause damage to surrounding tissues. However, the degree of swelling in the injured area will affect the length of time required for recovery [18]. Bleeding from injured blood vessels and exudation from injured tissue cells may affect wound healing. Therefore, it is particularly important for sports loss prevention. The way of prevention varies according to the possible losses caused by different sports, and there will be certain differences. For example, measures to prevent basketball sports injuries and the traditional prevention of basketball sports injuries mainly focus on warm-up before the game, relaxation after the game, and flexibility and strength training, but the emergence of functional training has brought new ideas to prevent sports injuries. The importance of the emphasized movement is considered to be "sport is movement," and the correct movement pattern is the key to preventing sports injuries. From this perspective, simply stretching and strengthening muscle strength will not change its original wrong movement habits, and bad movement habits are the culprit causing sports injuries. Therefore, in preventing sports injuries, it is necessary to improve joints. On the basis of flexibility and stability, further correcting the wrong action patterns or habits can truly reduce the incidence of sports injuries [8]. Through analysis, we have reached the following five conclusions about sports injuries.

First, the common injury sites and types of athletes will be different due to the sports characteristics of different events; second, most injuries occur during normal training. The main reason is insufficient warm-up activities and poor physical fitness; third, athletes wear protective gear or use sports tapes to prevent sports injuries; fourth, athletes reduce the occurrence of sports injuries through related preventive cognition concepts and emphasize the prevention of sports injuries ideologically and enhance the cognition concepts of sports injury prevention; and fifth, the attitude of athletes after injury is related to injury cognition. The higher the awareness of sports injury, the more positive their attitude will be, and the degree of injury will be minimized.

2.3. Fault Tree Analysis Method. There are many detection methods for athletes' injuries, but the traditional detection methods have small detection targets and the results are not very accurate. For this reason, this paper introduces fault tree analysis to optimize the layout of athletes' injuries detection [19].

Fault tree analysis technology integrates mathematical statistics, logical algebra, probability theory, optimization, graph theory, random processes, algorithmic complexity, and other mathematical branching sciences to study complex system problems. Applying fault tree analysis to carry out risk assessment research can realize the identification of risk factors in the system, measure the probability of accidents, and provide solutions for risk control. The fault tree is an inverted tree structure composed of a series of nodes and connections between nodes. Each specific node on the fault tree represents a certain event, and the connection between the nodes represents the relationship between the two events [20]. The events at the top of the fault tree are called top events, the events at the bottom of the fault tree are called cause events, and the events between the top event and cause events are called intermediate events in different chromatograms. The relationship between events may be and, or, and not. Generally speaking, there are two kinds of fault tree analysis, which are divided into downward method and upward method [3].

In the downlink method, logic OR gates are generally used to increase the number of fault tree cut sets, and logic AND gates are used to increase the capacity of fault tree cut sets. Starting from the analysis of the top event of the fault tree, the upper-level events are converted to the lower-level events in the order of the fault tree from top to bottom [21]. In a down method table, for logic AND gates, the input events for each logic AND gate are arranged horizontally. In the table, for logic OR gates, the input events for each logic OR gate are placed vertically in the table, and each logic gate is analyzed accordingly until the basic events in the fault tree are transformed. The last column of the table is the cut set of the fault tree which generally requires logical simplification to obtain the minimum cut set of the fault tree in actual analysis and calculation, as shown in Figure 1.

Through the step-by-step analysis of the downward method, the calculation steps are as follows:

$$
\min _{u \in B V} E(u)=a \int \frac{1}{p}|\nabla u|^{p} \mathrm{~d} x+(1-a) \int \frac{1}{q}|\nabla u|^{q} \mathrm{~d} x+\frac{\lambda}{2} \int|u-f|^{2} \mathrm{~d} x .
$$

This formula can be transformed into 


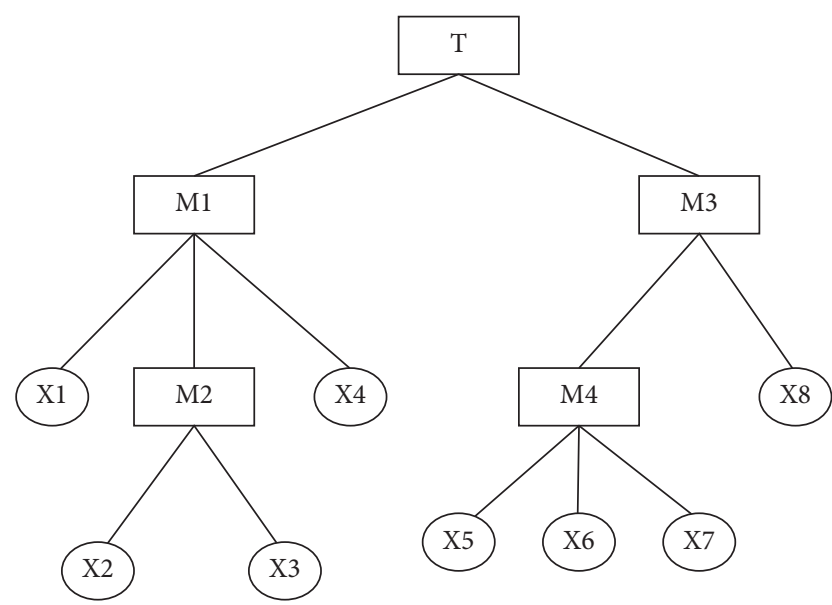

FIgURE 1: Schematic diagram of fault tree.

$$
N(x, y)=\sum_{k=1}^{n} w_{k} \exp \left(-\frac{\left\|V_{0}(x, y)-c_{k}\right\|}{2 \sigma_{k}^{2}}\right) .
$$

Weight adjustment admission is given by

$$
\begin{aligned}
\Delta w_{k} & =\sum_{x, y} \frac{\partial E(x, y)}{\partial w_{k}} \\
\Delta c_{k} & =\sum_{x, y} \frac{\partial E(x, y)}{\partial c_{k}} .
\end{aligned}
$$

Variants of nonlinear motion equations are as follows:

$$
\frac{a^{2} R}{\mathrm{at}^{2}}=b_{o}^{2}\left[1-2 \eta_{2} \frac{\mathrm{au}}{\mathrm{ad}}+\cdots\right] \frac{\mathrm{a}^{2} R}{\mathrm{ad}^{2}} .
$$

The solution of the equation is

$$
R=R_{1} \cos (q x-\mathrm{wd})-\frac{1}{4} \eta_{2} q^{2} R_{2} x \sin 2(\mathrm{qx}-\mathrm{wd})+\cdots
$$

In the ascending method, the fault tree is solved in the order from bottom to top. The logic gate symbols of some basic events in the fault tree are replaced with corresponding set operation symbols, and then the event symbols and set operation symbols are used for analysis according to the structure function of the fault tree. Calculate the number set of the fault tree, then use the set operation rules to logically simplify the obtained number set, and finally get the minimum number set of the fault tree [22]. The ascending method is used to solve the minimum cut set in the fault tree shown in the figure. The specific calculation steps are as follows.

The upper-level expression based on the event is given by

$$
\begin{aligned}
& M_{2}=x_{2} \cup x_{3}, \\
& M_{4}=x_{5} \cup x_{6} \cup x_{7} .
\end{aligned}
$$

The next level expression is

$$
\begin{aligned}
& M_{1}=x_{1} \cap M_{2} \cap x_{4}, \\
& M_{3}=M_{4} \cap_{x}^{8} .
\end{aligned}
$$

For a system fault tree with $n$ minimum cut sets $K_{j}(i=1,2, \ldots, n)$, if the basic events in each minimum cut set are not repeated and the basic events are independent of each other, the formula for calculating the probability of occurrence of the fault tree top event is as follows:

$$
P(x)=\sum_{j=1}^{n} \prod_{x_{i} \in K_{j}} q_{i} \text {. }
$$

If there are repeated basic events in each minimum cut set, then equation (8) is no longer valid. It is necessary to expand equation (8) to eliminate the repetition factor in the probability of each basic event according to the idempotence law of Boolean algebra. At this time, from the top event of the fault tree, the general formula of probability is given by

$$
P(x)=\sum_{j=1}^{n} \prod_{x_{i} \in K_{j}} q_{i}-\sum_{1 \leq j<h \leq n}\left(\prod_{x_{i} \in K_{j} \cup} K_{h} q_{i}\right)+\cdots+(-1)^{n-1} .
$$

The focus of the quantitative analysis of the fault tree is to calculate the importance of basic events. In the qualitative analysis of the fault tree of the system, many minimum cut sets can be obtained. At the same time, each minimum cut set is composed of multiple basic events. The probability of the occurrence of basic events leads to different functions of the top event failure, that is, the importance of the basic events of the fault tree is different [23]. The algorithm flow of the fault tree is shown in Figure 2.

In the system fault tree, the number of normal states of basic events is increased:

$$
y\left(k T+t_{i}\right)=\frac{1}{\alpha(z)} \sum_{j=1}^{r} \beta_{i j}(z) \bar{u}\left(k T+t_{j-1}\right)+v\left(k T+t_{i}\right) .
$$

It can be transformed into

$$
\begin{gathered}
\alpha(z)=1+\alpha_{1} z^{-1}+\alpha_{2} z^{-2}+\cdots+\alpha_{n} z^{-n}, \\
\beta_{i j}(z)=\beta_{i j}^{0}+\beta_{i j}^{1} z^{-1}+\beta_{i j}^{2} z^{-2}+\cdots+\beta_{i j}^{n} z^{-n} .
\end{gathered}
$$

Its function is to move the sampling signal $s\left(k T+t_{i-1}\right)$ in time backward $s\left(k T+t_{i-1}\right)$ by 1 nonuniform sampling interval, and a new transfer function model is proposed:

$$
y\left(k T+t_{t}\right)=\frac{B_{i}(\delta)}{A_{i}(\delta)} \bar{u}\left(k T+t_{i}\right)+v\left(k T+t_{i}\right) .
$$

\section{Experiments on the Statistical Calculation Method of Sports Injuries}

3.1. Subjects. The research object of this study is the cognition and prevention of sports injuries of athletes in a sports university team in this city. The subjects of this study are athletes from a sports university team. There are a total of 8 


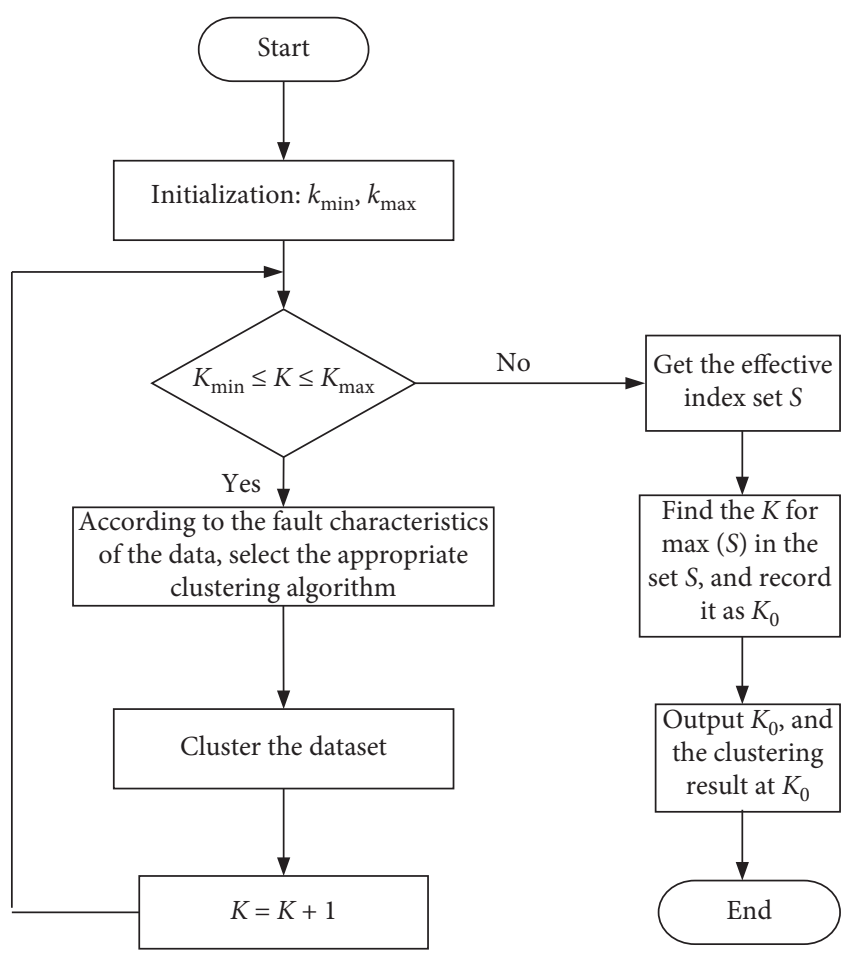

FIGURE 2: Fault tree process analysis algorithm.

teams in the school team list announced by the Competition Office of the University of Sports. They are basketball, volleyball, football, shuttlecock, track and field, swimming, martial arts, and table tennis.

\subsection{Establishment of a Model Evaluation Index System.} By actually observing the object, you can draw clear conclusions. In general, a metric system contains three levels of metric that are related to gradual decomposition and improvement. Among them, the first-level evaluation index and the second-level evaluation index are relatively abstract and cannot be used as a direct basis for evaluation. The thirdlevel evaluation indicators should be specific, measurable, and behavior-oriented and can be used as a direct basis for evaluation. We make statistics on these athletes, as shown in Table 1 .

3.3. Determination of the Evaluation Weight. The index weight is a numerical index indicating the importance and function of the index. In the indicator system of the evaluation plan, the weight of each indicator is different. Even if the indicator level is the same, the weight is different. Index weight is also called weight and is usually represented by $a$. It is a number greater than zero but less than 1, and the sum of the weights of all first-level indicators must be equal to 1 , that is, satisfying the conditions $0<a<1$ and $\sum a-1$.

3.4. Statistics. All data analysis in this article uses SPSS19.0, statistical test uses two-sided test, significance is defined as 0.05 , and $p<0.05$ is considered significant. The statistical results are displayed as mean \pm standard deviation $(x \pm \mathrm{SD})$.
When the test data follow a normal distribution, the double $T$ test is used for comparison within the group, and the independent-sample $T$ test is used for comparison between the groups. If the regular distribution is not sufficient, two independent samples and two related samples will be used for inspection.

\section{Experimental Analysis of Statistical Calculation Methods for Sports Injuries}

4.1. Current Status of Athletes' Injuries. We conducted statistics on selected athletes related to sports injuries and divided the human body into four parts: head and neck, trunk, upper limbs, and lower limbs. Statistics on different parts of the injury are shown in Table 2.

From Table 2, we can see that the highest proportion of injured parts is the lower extremity $(52.1 \%)$, including knees (21.8\%, 55 people), ankles (18.7\%, 47 people), thighs $(5.2 \%$, 13 people), toes ( $4 \%, 10$ people), calf (1.2\%, 3 people), and Achilles tendon (1.2\%, 3 people). The second largest physical injury is the upper extremity, which includes wrists $(11.9 \%$, 30 people), shoulders $(10.7 \%, 27$ people), fingers $(4 \%, 10$ people), and elbows (2\%, 5 people). The third place in physical injury is the trunk, which includes the lower back (11.1\%, 28 persons), the upper back (1.6\%, 4 persons), and the chest and abdomen $(0.4 \%, 1$ person). The fourth largest physical injury is the head and neck: neck $5.2 \%$ (13 people) and head $1.2 \%$ (3 people).

According to the different parts of the injury, we will make statistics on the injury situation of the athletes in different sports, as shown in Figure 3.

It can be clearly seen from Figure 3 that, due to different parts of the body that need to be used for different sports, athletes will suffer different sports injuries during training. For example, in swimming, the most commonly injured part of an athlete is the back. In ball games, the upper and lower limbs are the most commonly injured, accounting for more than 60 people injured in ball games. The author explained that when we study sports injuries, we must distinguish them according to different sports.

4.2. Types of Damage. We classify damage according to different types of damage. Sprain refers to the torsion or damage of the ligament due to twisting or overextension; strain refers to abnormal muscle activity or sudden huge contraction, causing muscle fiber to break; and contusion refers to bone or muscle suffering. The impact of external force caused the rupture of blood capillaries and muscle fibers, and the redness and swelling of internal hemorrhage occurred in the injured area. Specific statistics are shown in Table 3.

As shown in Table 3, according to the statistics of the injury survey of athletes, the ranking of injury types is as follows: sprains accounted for $44 \%$ (112 people), strains accounted for $31 \%$ (77 people), contusions accounted for $16 \%$ (41 people), bruises accounted for $4.8 \%$ (12 persons), fractures accounted for $2 \%$ (6 persons), dislocations accounted for $0.8 \%$ ( 2 persons), and cut injuries accounted 
TABLe 1: Statistics of college athletes.

\begin{tabular}{lcc}
\hline & Number of people & Percentage \\
\hline Male & 132 & 53.8 \\
Female & 113 & 46.2 \\
Freshman & 92 & 37.5 \\
Sophomore & 62 & 25.3 \\
Junior & 59 & 24.1 \\
Senior year & 32 & 13.1 \\
\hline
\end{tabular}

TABLE 2: Injury status of athletes.

\begin{tabular}{lcc}
\hline & Number of people & Proportion (\%) \\
\hline Head & 3 & 1.2 \\
Neck & 13 & 5.2 \\
Chest and abdomen & 1 & 0.4 \\
Back & 32 & 12.7 \\
Shoulder & 27 & 10.7 \\
Elbow & 5 & 2.0 \\
Wrist & 30 & 11.9 \\
Finger & 10 & 4.0 \\
Thigh & 13 & 5.2 \\
Knee & 55 & 21.8 \\
Calf & 3 & 1.2 \\
Achilles tendon & 3 & 1.2 \\
Ankle & 47 & 18.7 \\
Toe & 10 & 4.0 \\
\hline
\end{tabular}

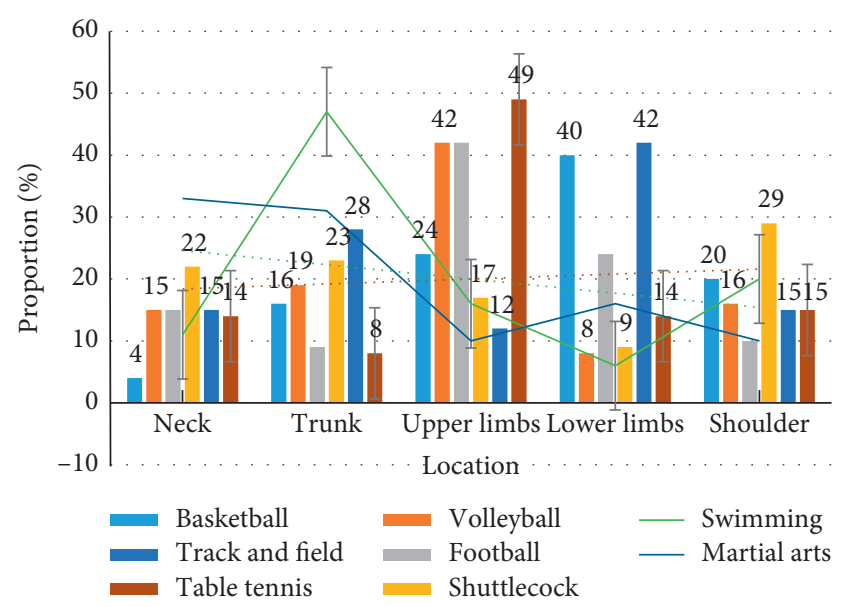

FIGURE 3: The proportion of different parts of the damage.

for $1.5 \%$ (4 persons). We have made statistics on the types of injuries in different sports, as shown in Figure 4.

As shown in Figure 4, sports injuries caused by different sports are different. Sports such as basketball and football can easily cause athletes to sprain and strain, while sports such as swimming and track and field can easily cause abrasions and dislocations. In general, in the current statistics of college sports, the most common sports injuries suffered by athletes are sprains, strains, and abrasions.

4.3. Causes of Injury and Prevention. In the survey, it was found that the timing of sports injuries occurred in $67.5 \%$ (170 people) in normal practice, $15.5 \%$ in prematch practice
(43 people), and $17.1 \%$ (43 people) in competitions. During the preparation period, due to the increase in exercise intensity, the number of repetitions increases, the requirements for physical fitness and ability during training increase, and the exercise load increases, which can easily cause acute injuries. Therefore, the rate of injury is the highest during this period, as shown in Table 4.

Athletes' training plans should be arranged according to the differences of different individuals. The training course arrangement should fully reflect its diversity, comprehensiveness, pertinence, and gradualness, avoid long-term monotonous and rigorous training methods, and follow the coach's guidance, step-by-step training courses, and training staging plans to avoid overtraining. At present, the main causes of sports injuries are insufficient preparation activities, accidents, overtraining, etc. We have made statistics on them, as shown in Figure 5.

It can be seen from Figure 5 that there are many causes of sports injuries. The common causes of sports injuries are listed in order for investigation: inadequate preparation, accidents, overtraining, technical errors, carelessness, weakness, unfamiliar movements, poor venue facilities, etc. The main purpose of preexercise preparation activities is to increase the heart rate and promote blood circulation. Warm-up activities will increase the body temperature and stimulate blood vessels to dilate, increase the local blood flow in the active part, and reduce the possibility of sports injuries.

We divide athletes into men and women, understand their understanding of sports injuries in recent years, and make statistics on their changes, as shown in Figure 6.

As shown in Figure 6, there is little difference in the understanding of sports injuries between male and female athletes, but overall, the understanding is not deep, and the degree of change is not large. However, in recent years, due to the popularization of sports knowledge, more attention has been paid to the content of sports injuries, so the degree of understanding has also increased in recent years.

When the injury occurs, the control of the inflammatory response in the acute phase will affect the speed of recovery. Ice can lower the temperature of the tissues and the tissues produce vasoconstriction, thereby reducing tissue bleeding; it can also slow down cell metabolism, cells are stimulated by low temperature, and oxygen demand will be significantly reduced, which can greatly reduce the metabolism of the injured area. The function of hot compress is to promote metabolism, increase the elasticity and softness of soft tissues, and promote tissue healing. Hot compresses are usually used 72 hours to six weeks after injury to repair soft tissues. Through investigation, we understand the athletes' handling methods, as shown in Figure 7.

It can be seen from Figure 7 that, after a sports injury, athletes will mainly use hot compresses and ice compresses for treatment, but due to the different parts of the injury, the correct treatment methods will also change. The author also explained that our city's knowledge about sports injuries is still insufficient. In the future sports education process, we should pay attention to the acceptance of sports injuries and reduce the situation of sports injuries. 
Table 3: Percentage of different types of damage.

\begin{tabular}{lcc}
\hline Type of damage & Number of people & Proportion (\%) \\
\hline Sprain & 112 & 44 \\
Strain & 77 & 31 \\
Dislocation & 2 & 0.8 \\
Fracture & 6 & 2 \\
Contusion & 41 & 16 \\
Bruise & 10 & 4.7 \\
Cut wound & 4 & 1.5 \\
Puncture wound & 0 & 0 \\
\hline
\end{tabular}

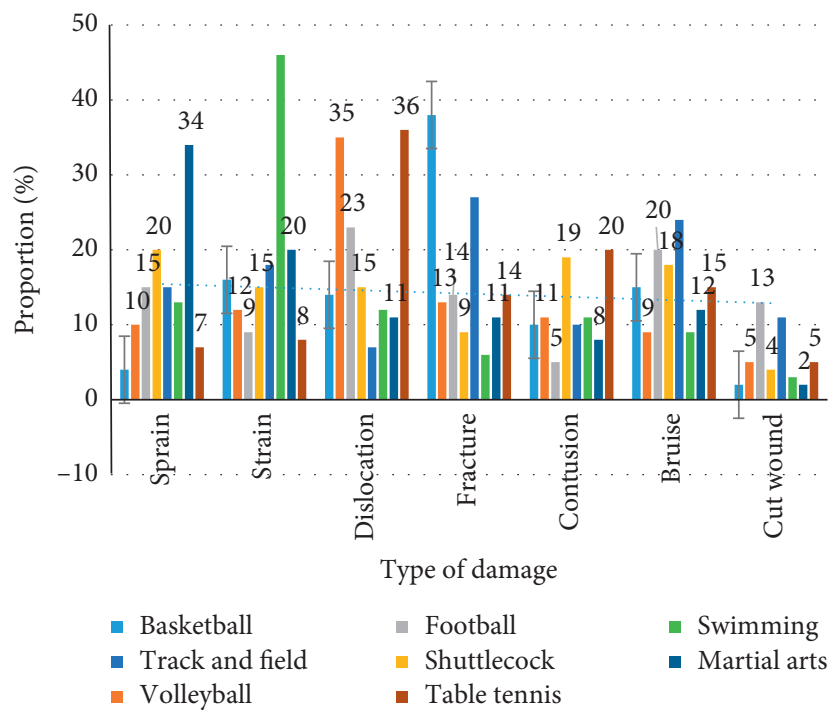

Figure 4: Percentage of damage types.

Table 4: Timing of injury.

\begin{tabular}{lcc}
\hline Timing of injury & Number of people & Proportion (\%) \\
\hline In the game & 43 & 17.1 \\
Prematch practice & 39 & 15.5 \\
Usual practice & 170 & 67.5 \\
\hline
\end{tabular}

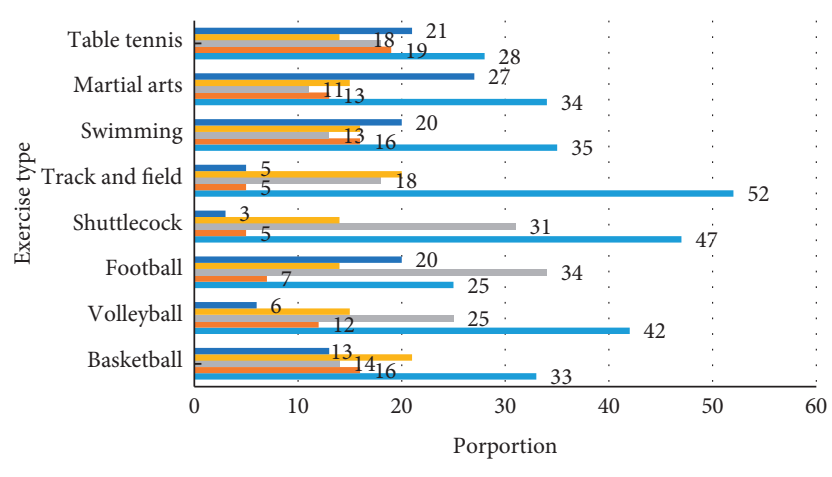

\footnotetext{
- Overtraining $\quad$ Unfamiliar movements

- Lack of preparation - Venue and equipment

-1. Poor physical fitness
}

Figure 5: Main causes of damage. 


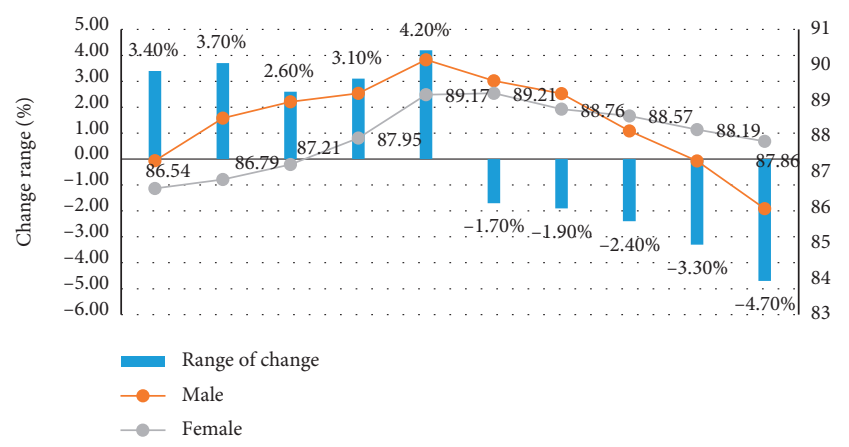

FIGURE 6: Changes in the understanding of sports injuries in recent years.
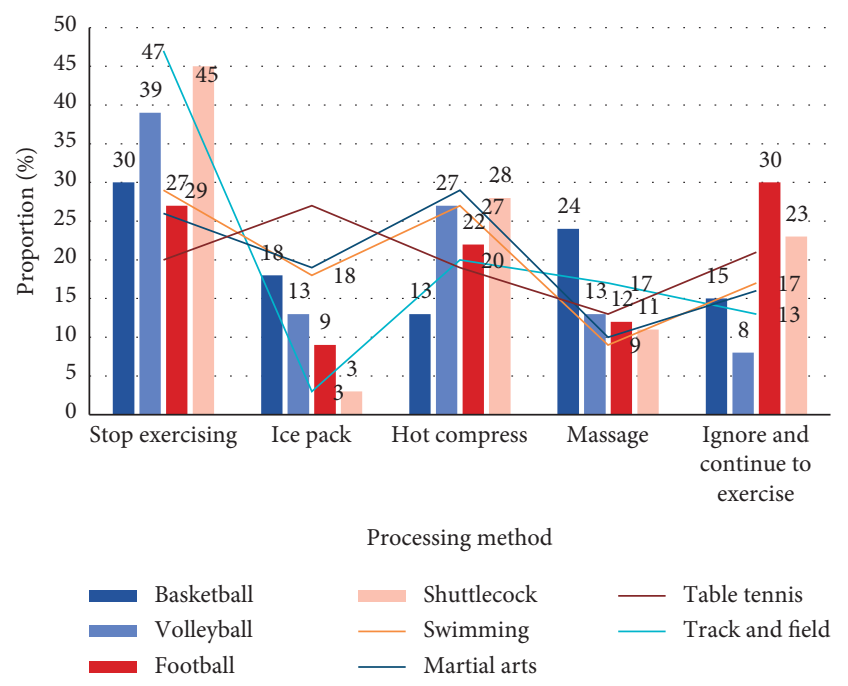

FIgURE 7: Sports injury treatment.

\section{Conclusion}

Pay attention to the prevention of sports injuries. The occurrence of sports injuries is closely related to the lack of relevant knowledge of coaches and athletes. Athletes lack awareness and understanding of general sports injuries and lack the theoretical knowledge to deal with injuries when they occur. After the injury, the injury is not clarified. The cause of the occurrence is likely to cause the injury to occur again and cause secondary injury to the injured part. In order to improve athletes' knowledge about injuries, coaches can emphasize the harm caused by sports injuries and pay attention to safety education from daily training, so that athletes can pay attention to the prevention of sports injuries in their ideology and strengthen their concepts of injury prevention. In the training process, combined with actual operating experience, let the athletes be familiar with the methods of dealing with injuries. At the same time, the prevention-oriented training policy should be strengthened to correct athletes' wrong exercise patterns and wrong postures and train for weak muscle groups, which can reduce the internal factors that cause injuries and achieve the effect of preventing injuries. There are also some shortcomings in the research of this paper. Due to time and funding, the sample selected in this paper is not comprehensive enough, personal considerations are not complete enough, the mastery of measurable variable data is not comprehensive, the measurable variables determined for related latent variables are not comprehensive enough and cannot fully reflect the influencing factors of sports injuries, and the analysis results obtained are not very satisfactory. In the future research process, it is necessary to comprehensively consider and increase the width of the sample and time so that the results of the experiment can be more perfect.

\section{Data Availability}

No data were used to support this study.

\section{Conflicts of Interest}

The author declares that there are no conflicts of interest.

\section{References}

[1] H. Dhillon, S. Dhillon, and M. S. Dhillon, "Current concepts in sports injury rehabilitation," Indian Journal of Orthopaedics, vol. 51, no. 5, pp. 529-536, 2017.

[2] I. N. Ackerman, M. A. Bohensky, J. L. Kemp, and R. de Steiger, "Likelihood of knee replacement surgery up to 15 years after sports injury: a population-level data linkage study," Journal of Science and Medicine in Sport, vol. 22, no. 6, pp. 629-634, 2019.

[3] M. Casals and R. O. Nielsen, "Who and what can contribute to improve the statistical thinking in sports injury research? a humorous analogy between basketball and members of the multidisciplinary research team," Apunts. Medicina de l'Esport, vol. 54, no. 203, pp. 81-84, 2019.

[4] Z. Y. Kerr, S. L. Zuckerman, J. K. Register-Mihalik et al., "Estimating concussion incidence using sports injury surveillance systems," Neurologic Clinics, vol. 35, no. 3, pp. 409-434, 2017.

[5] D. Forsdyke, A. Smith, M. Jones, and A. Gledhill, "Infographic: psychosocial factors associated with outcomes of sports injury rehabilitation in competitive athletes," British Journal of Sports Medicine, vol. 51, no. 7, p. 561, 2017.

[6] J. Zhu, B. Arsovska, and K. Kozovska, "Acupuncture treatment for sports injury-hamstring muscles group," International Journal of Clinical and Experimental Medical Sciences, vol. 3, no. 6, pp. 71-73, 2017.

[7] J. S. Everhart, K. Harris, A. Chafitz et al., "Psychological assessment tools utilized in sports injury treatment outcomes research: a review," Journal of Sports Science \& Medicine, vol. 19, no. 2, pp. 408-419, 2020.

[8] H. Wang and N. Liu, "Research on the examination value of high-frequency ultrasound in the postoperative rehabilitation treatment of sports injury achilles tendon rupture," Journal of Medical Imaging and Health Informatics, vol. 10, no. 4, pp. 918-922, 2020.

[9] M.-Y. Chen, Y.-L. Kuo, and C.-Y. Chou, "Lateral abdominal muscle symmetry and risk of sports injury in baseball players: 351 board \#167 May 27 10:30 AM-12:00 PM," Medicine \& Science in Sports \& Exercise, vol. 52, no. 7S, p. 83, 2020.

[10] C. L. Udby, F. M. Impellizzeri, M. Lind, and R. Ø. Nielsen, "How has workload been defined and how many workload-related exposures to injury are included in published sports injury articles? 
a scoping review," Journal of Orthopaedic \& Sports Physical Therapy, vol. 50, no. 10, pp. 538-548, 2020.

[11] T. A. Akhadov, R. A. Keshishyan, S. A. Valiullina, O. V. Bozhko, A. V. Manzhurtsev, and M. V. Ublinskiy, "Magnetic resonance imaging of a sports injury of the lower extremities in children," Russian Pediatric Journal, vol. 23, no. 4 , pp. 235-239, 2020.

[12] C. Gho, J. L. Petro, and D. A. Bonilla, "Influencia de la acupuntura sobre el tratamiento de lesiones deportivas y el rendimiento atlético: una revisión integradora," Revista Internacional de Acupuntura, vol. 14, no. 2, pp. 64-75, 2020.

[13] B.-J. Juan de Dios, A. L. Garrigosa, P. D. Cuevas et al., "Translation into Spanish and proposal to modify the orchard sports injury classification system (OSICS) version 12," Apunts Sports Medicine, vol. 55, no. 207, pp. 105-109, 2020.

[14] X. Li, Y. Wang, and G. Liu, "Structured medical pathology data hiding information association mining algorithm based on optimized convolutional neural network," IEEE Access, vol. 8, no. 1, pp. 1443-1452, 2020.

[15] R. D. Comstock and S. K. Fields, "The eternally wounded athlete: how medical professionals and sports injury researchers have limited female athletes' sport participation and biased the interpretation of sports injury research," Current Epidemiology Reports, vol. 7, no. 4, pp. 327-333, 2020.

[16] G. S. Dharmshaktu, "Sternum fracture: an uncommon sports injury," Saudi Journal of Sports Medicine, vol. 19, no. 1, pp. 28-29, 2020.

[17] M. Elhoseny, G.-B. Bian, S. K. Lakshmanaprabu, K. Shankar, A. K. Singh, and W. Wu, "Effective features to classify ovarian cancer data in internet of medical things," Computer Networks, vol. 159, pp. 147-156, 2019.

[18] H. Ba, "Medical sports rehabilitation deep learning system of sports injury based on MRI image analysis," Journal of Medical Imaging and Health Informatics, vol. 10, no. 5, pp. 1091-1097, 2020.

[19] A. Kondo, C. Ishioka, and N. Hongu, "Sports injury management program in pima community college offers study abroad opportunities for international students," Medicine \& Science in Sports \& Exercise, vol. 51, pp. 990-993, 2019.

[20] K. W. Young, K. C. Park, J. S. Hwang, and H. S. Lee, "Peroneal tendon repair in sports injury," Journal of Korean Foot and Ankle Society, vol. 23, no. 3, pp. 100-103, 2019.

[21] K. Geetha, V. Anitha, M. Elhoseny, S. Kathiresan, P. Shamsolmoali, and M. M. Selim, "An evolutionary lion optimization algorithm-based image compression technique for biomedical applications," Expert Systems, vol. 38, no. 2, 2020.

[22] C. Ortiz, "Guest editorial: innovations in foot and ankle sports injury treatment," Techniques in Foot \& Ankle Surgery, vol. 18, no. 2, p. 49, 2019.

[23] Q. Lan and X. Li, "Liability determination of school sports injury accidents: an analysis framework based on evolutionary game," International Journal of Environmental Research and Public Health, vol. 16, no. 18, pp. 3403-3406, 2019. 